\title{
Oxane Leingang
}

Institut für Kinderbuchforschung der Johann Wolfgang Goethe-Universität, Frankfurt am Main leingang@em.uni-frankfurt.de

\section{Fiktionalisierung der eigenen Kriegskindheit in den Jugendromanen von Anatolij Pristavkin}

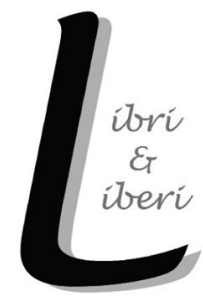

„,Mich erschuf der Krieg“, notierte Anatolij Pristavkin in seinem autobiographischen Werk Der erste Tag - der letzte Tag der Schöpfung, worin der Krieg für ihn tatsächlich zu einer Sozialisierungsinstanz wurde. Pristavkins Mutter starb 1941 an Tuberkulose, als er zehn Jahre alt war; der Vater kämpfte an der Front. Im Jahre 1944 wurde er zusammen mit mehreren hundert Moskauer Waisen in den Nordkaukasus evakuiert, wo er als einer von wenigen einem Massaker entgehen konnte. Aus Trauer um die eigene Kriegskindheit schrieb Pristavkin ein literarisches Großprojekt nieder. In sechs autobiographisch gefärbten, detailreichen Kurzromanen sowie in unzähligen Kurzgeschichten näherte sich der Autor dem Trauma seiner Kriegskindheit an. Da er dabei keine Autobiographiesignale im Sinne des ,, autobiographischen Paktes “ setzte und die Möglichkeit des „,moderaten Fiktionalisierungsschubs" nutzte, überführte er das biographisch Unartikulierte durch nachträglich imaginierte Entlastungsmomente in ,Wunschbiographien“. Im Folgenden wird untersucht, wie Anatolij Pristavkin die eigenen traumatischen Erinnerungen an den Zweiten Weltkrieg in seinen jugendliterarischen Werken inszenierte.

Schlüsselwörter: Autobiographie, Pristavkin, Kriegskindheit, Zweiter Weltkrieg, Jugendromane

\section{Werke Pristavkins im Kontext (post-)sowjetischer Kriegsliteratur}

Die schwierige Kindheit (Trudnoe detstvo) heißt der Zyklus von Kurzerzählungen über seine Kriegskindheit, mit dem Pristavkin 1959 debütierte. 
Dabei handelt es sich um kurze Bestandsaufnahmen des Lebens im Waisenhaus, in denen bereits Motive der späteren Werke intoniert werden, jedoch ohne deren desperate Essenz anzudeuten oder ein Mosaik zu ergeben. Dem zentralen autobiographischen Trauma entsprechend kreist seine autobiographische Serie um die Waisenkinder, das Strandgut der Säuberungswellen und des Krieges. Damit greift Pristavkin eine Motivkonstante auf, die seit den 1920er Jahren in der sowjetischen Kinder- und Jugendliteratur an Bedeutung gewann. Dass dieses Thema häufig behandelt wurde, ist geschichtlich bedingt: Hatten schon während des Ersten Weltkrieges und im Bürgerkrieg Tausende ihre Angehörigen verloren, so ließ der Terror der Stalin-Ära mit der landwirtschaftlichen Kollektivierung und den groß angelegten Säuberungen die Zahl der Waisen noch weiter in die Höhe steigen (vgl. dazu Snyder 2010: 43-58). Mit dem Beginn des Zweiten Weltkrieges schließlich waren sowjetische Städte voll von heimatlosen Kindern, die ihre Familien im allgemeinen Tumult der unionsweiten Evakuierungen oder im Krieg verloren hatten.

Die parteitreuen Autoren, die das Thema der Waisen darlegten, zollten der bolschewistischen Weltanschauung Tribut und ließen ihre verwahrlosten Helden unter dem Einfluss von überzeugten Kommunisten zu geläuterten Sowjetmenschen resozialisieren. ${ }^{1}$ Im Gegensatz zu seinen Vorgängern setzt Pristavkin den Akzent auf die literarische Anklage: Indem Pristavkin die verordnete Brutalität im totalitären System darstellt, enthüllt er die Doppelmoral der sowjetischen Gesellschaft und hinterfragt ihre Feindkonzeption, die sich gegen elternlose Kinder wendet. Auch wenn Kinder in seinen Werken die Hauptakteure von betrügerischen Machenschaften sind, verkörpern sie Menschlichkeit, Unschuld und Integrität. Mit seinen Werken demontiert er die Hauptaspekte des sowjetischen Kindheitskonzepts, die in der (Kinder- und Jugend-)Literatur und Propaganda heraufbeschworen wurden - die moralische Erziehung und den Mythos von der glücklichen Kindheit (vgl. dazu Knight 2009: 790-808). Wie alle Kinder wurden insbesondere die Heimkinder indoktriniert, dem sowjetischen Staat, der sie vor dem Elend bewahrt hat, Dankbarkeit und Loyalität entgegenzubringen: Sie hätten das Glück gehabt, in dem „besten Land der Welt“ geboren zu sein (vgl. dazu Kelly 2007: 93-108). In seinen Kindheitserinnerungen wendete sich Pristavkin gegen die „Verkleisterung der Geschichte durch Pathos und Sentimentalität“ (Jureit 2010: 34). Erinnert werden soll nicht nur an erlittene, sondern auch an zu verantwortende Verbrechen, wie den brutalen Umgang mit

${ }_{1}$ Als das bekannteste Buch zu dieser Thematik gilt Respublika Škid (1926/27), der autobiographisch gefärbte Roman des Autorenduos Leonid Panteleev und Grigorij Belych, in dem verwahrloste Jugendliche erfolgreich in die sozialistische Gesellschaft integriert werden. 
verwahrlosten Kindern, deren Eltern dem ,Reinigungsmechanismus' des Regimes oder dem Krieg zum Opfer gefallen waren.

Pristavkins Erstlingswerk fiel in die Phase der Privatisierung von Kriegserinnerung. Mit der Neubewertung der Stalinzeit im Jahre 1956 konnte sich auch die Kunst teilweise aus dem Korsett des sozialistischen Realismus befreien (vgl. Warneke 2000: 43). Das propagandistisch Monumentale der Kriegs- und Nachkriegszeit wurde zugunsten einer Psychologisierung des Protagonisten, meist eines einfachen Soldaten, aufgegeben. Die Kulissen waren nicht mehr strategisch wichtige, sondern unbedeutende Frontabschnitte. Die heroischen und panoramischen Elemente schwanden, während das Tragische des Einzelschicksals in den Mittelpunkt rückte. Auch die Ästhetik verlor ihre Uniformität. Als Folge dieser Entwicklung entstand die so genannte ,Leutnantsliteratur', in der die einstigen blutjungen Soldaten ihre Fronterfahrungen schilderten. So wundert es kaum, dass regimetreue Kritiker an dieser ,Schützengrabenwahrheit' den scheinbar dysfunktionalen Detailreichtum monierten. Nach dem Zusammenbruch der Sowjetunion fand in den Aufzeichnungen der damaligen Kriegskindergeneration eine weitere Emotionalisierung der Geschichte statt, die nicht nur zum post-heroischen Paradigmenwechsel beitrug, sondern den Akzent erneut vom Uniformierenden hin zum Individuellen, von der Helden- zur Opfersemantik verschob.

\section{Sowjetisierung der westeuropäischen Klassiker als text- internes Phänomen}

In den Werken Pristavkins wird mehrmals das Moment der fiktiven Überformung thematisiert. Das Fehlen der Gewissheit über die eigene Herkunft - einer Komponente sowohl des Selbstbewusstseins als auch des sozialen Status - führt dazu, dass seine Helden sich in eskapistischen Träumen eine glamouröse Biographie zusammenfabulieren. Insbesondere das Bild des fremden Vaters, der in den Kindern Stolz und Sehnsucht erregt, dient als attraktive Projektionsfläche für heroische Phantasien. So werden alle Väter als Generäle oder zumindest als hochdekorierte Kriegshelden idealisiert.

Die Waisen Pristavkins verändern auch westeuropäische Werke: In Scheherezade-Manier werden Romane von Dumas, Hugo, Dickens und Twain in den Kinderheimen nacherzählt. Auf der Metaebene der Erzählgegenwart sind die intertextuellen Verzahnungen durch die Nennung des Verfassers oder des Werkes explizit markiert. Gerade weil die kindlichen Erzähler Nacht für Nacht mit einer Kruste Brot belohnt werden, sind die kanonischen Werke der Abenteuerliteratur fiktiv überformt und gedehnt; die burlesken Parodien sollen die kindlichen Zuhörer 
in Wirbel von fesselnden Abenteuern verstricken. Vor dem Erfahrungshorizont der Waisen werden alle Werke adressatenorientiert in ihre derbe Sprache übersetzt und kontextabhängig in die sowjetische Realität transponiert: Der Gascogner d'Artagnan rächt sich beispielsweise an den Peinigern, indem er sie nach einer halsbrecherischen Verfolgungsjagd zur Strecke bringt. Der Lausbub Tom Sawyer ist ein erfolgreicher Taschendieb auf einem der Moskauer Märkte, wo er regelmäßig seine Freundin, die Eisverkäuferin Becky, trifft. Auf dem Roten Platz peitscht der Graf von Monte Christo den Direktor eines Waisenheims aus (vgl. Pristavkin 2006: 481). Die vorgenommene Erweiterung des Themenspektrums, der Vertrautheitsaspekt der Lebenswelt zusammen mit den Elementen der westeuropäisch-exotischen Abenteuergeschichten machen die Attraktivität dieser Klassiker-Adaptionen aus. ${ }^{2}$

Die Spannung zwischen Fiktionalität und Authentizität lässt sich nur mit Kontextwissen zur Biographie Pristavkins auf der text-externen Ebene erkennen. Um deren Funktionsmechanismen und Wirkungspotentiale zu beleuchten, stelle ich in folgendem Abschnitt exemplarisch einige narrative Anreicherungen vor.

\section{Fiktionale Anreicherung des autobiographischen Kerns}

Der Soldat und der Junge (Soldat $i$ malč̀k) von 1970/1971 bildet den Auftakt seiner durch das Figurenensemble lose verklammerten Trilogie über die Kriegskindheit. Diese Erzählung schildert eine ungewöhnliche Freundschaft zwischen einem jungen Soldaten und einem Waisenkind. Ein traumatisches Erlebnis im Kinderheim lieferte das Handlungsgerüst für das konfliktreiche Miteinander, verriet Pristavkin in einem Interview. ${ }^{3}$ Die Erinnerung an die Tyrannei im Kinderheim wird in dieser povest' abweichend von der eigenen Biographie durch ein konstruiert wirkendes Happyend überformt. Gerade die Kenntnis der tatsächlichen Begebenheit wirkt als eine Kontrastfolie für die neue Schlussvariante: Während der Protagonist Waska einem Jungkriminellen Paroli bietet, musste der junge Anatolij Pristavkin überstürzt aus dem Kinderheim fliehen. Mit nachträglich hinzuerfundenen entlastenden Zwischenspielen funktionalisiert der Autor seine

2 Zum Verfahren der thematischen Akkomodation des allgemeinliterarischen Stoffrepertoires vgl. Ewers 2012: 184-190.

3 „Es passierte während des Krieges. Auf der Bahnstation Tomilino bei Moskau haben ein paar Burschen, mich eingeschlossen, einen schlafenden Soldaten bestohlen. Und dann kam er in unser Kinderheim. Und so passierte es, dass ich ihn auf der Suche nach den anderen, die in den Diebstahl verwickelt waren, von Haus zu Haus führte. Die Reaktion der Mitmenschen war unterschiedlich; aber manche hatten mir offen gedroht, wenn ich mit der Suche nicht aufhören sollte. Tatsächlich haben sie mir dann aufgelauert und mich zusammengeschlagen. Um mein Leben zu retten, flüchtete ich nachts und ohne Kleidung aus dem Kinderheim. In jener Zeit war das Bestehlen eines unbekannten Soldaten und die anschließende Flucht keine Ausnahme. Und ich hatte es schnell vergessen." (Kardin 2000) 
Selbstinszenierung und therapiert die seelischen Verwundungen literarisch durch eine sympathetische Wendung an das ,innere Kind'.

Schlief ein goldenes Wölkchen (Nočevala tučka zolotaja), das seinem Autor den größten Ruhm und die höchste Auflagenzahl brachte, konnte wegen der Zensur erst ein Jahrzehnt später erscheinen. ${ }^{4}$ Die ausgehungerten Moskauer Waisenkinder Saschka und Kolka Kuzmin, die Helden des Werkes, werden im Frühjahr 1944 in den Kaukasus gebracht, während die dort ansässige tschetschenische Bevölkerung als ,illoyale Nationalität' nach Sibirien verschleppt wird. Im Kaukasus geraten die Zwillinge in einen Strudel blutiger Ereignisse. Die fiktionale Halbierung eines Individuums in Schlief ein goldenes Wölkchen macht die narrative Reorganisation der Erinnerungen offenkundig. Pristavkin verteilt seine persönlichen Erlebnisse im Nordkaukasus auf die Zwillingsbrüder. Diese Zerlegung des Ich lässt sich als eine dissoziative Wesensspaltung lesen - wie sie für traumatisierte Menschen typisch ist. In gegensätzliche Charakterzüge aufgeteilt, ergänzen sich die Kuzmin-Brüder zum beiderseitigen Nutzen und Schaden. Ihre frappierende physische Ähnlichkeit eröffnet ein weites Feld für heitere Verwechslungen und gibt dem Werk einen pikaresken Anstrich. Gerade diese anekdotischen Episoden dämpfen die Tragik der individuellen Schicksale.

Der Roman spielt mit verschiedenen Entwürfen einer homodiegetischen Erzählerinstanz: Beim Schreiben wiederholt sich die Spannung zwischen dem sich erinnernden und dem erinnerten Ich in Ich-Texturen, im komplizierten Verhältnis einer sich selbst erzählenden und von sich selbst erzählenden Figur. Im Handlungsverlauf pendelt die Erzählerstimme, die für das fünfhundertköpfige Kinderkollektiv spricht, zwischen der ersten Person Plural und der dritten Person Singular. Dieser Perspektivenwechsel als Verfahren der Fiktionalisierung ist dem Formenarsenal des Romans entnommen. Erst am Ende wird deutlich, dass die Hauptfigur Kolka und der Erzähler identisch sind. Der Tod eines der Brüder - der tragische Höhepunkt des Buches - steht für den symbolischen Tod des kindlichen Ich: „Ich hatte den Tod gesehen, hatte Erschießungen miterlebt, ich selbst war schon viele Male gestorben", so Pristavkin in einem Interview (vgl. Pristavkin 1991: 3931). Der Überlebende wird zum Stifter und Träger der Erinnerung.

${ }_{4}$ In den folgenden Jahren avancierte der Schriftsteller und Essayist Pristavkin zum führenden Menschenrechtler des Landes. Während des Ersten Tschetschenien-Krieges (1991-1996) reiste Pristavkin zweimal in das Krisengebiet, wo er Zeuge von Gewalttaten gegen die Zivilbevölkerung wurde und sie später in zahlreichen Vorträgen und Interviews publik machte. Von 1992 bis 2001 leitete Pristavkin die Kommission zur Begnadigung von Häftlingen, die zum Tode verurteilt werden sollten. Mit großer öffentlicher Anteilnahme wurde er am 14. Juli 2008 am Troekurov-Friedhof in Moskau beerdigt. Als langjähriger Berater des Präsidenten erhielt Pristavkin ein Staatsbegräbnis, das den zeitlebens härtesten Regimekritiker für die postsowjetische Heroengalerie vereinnahmt. Kurz nach seinem Tod erhielt eine Straße in der tschetschenischen Stadt Gudermes seinen Namen. 
Das abschließende Werk seiner Trilogie über Waisenheimkinder erschien 1989. Wir Kuckuckskinder oder ein Klagelied zur Beruhigung des Herzens (Kukušata ili žalobnaja pesn'dlja uspokoenija serdca), nach eigenen Aussagen das Lieblingsbuch des Autors (Pristavkin 2007: 486), schildert das Leben in einem „Spez“, einem Sonderheim, in dem Kinder von Opfern Stalinscher Verfolgungen unter fremden Namen untergebracht sind. Der Ich-Erzähler Sersch präsentiert eine psychologisch durchformte, mit Sympathie dargestellte Porträtgalerie der neun Waisenkinder, die man als Kukuschkin chiffriert hat. Die Anspielung auf die brutparasitäre Vogelart Cuculidae problematisiert die Abstammung der Kinder zusätzlich. Als die Waisen von ihrer wahren Herkunft erfahren, rebellieren sie gegen Niedertracht und Willkür und werden von der Miliz erschossen. So lässt sich dieser Roman als eine säkularisierte Märtyrerpassion lesen; der Tod der Unschuldigen gehört zum festen Bestandteil dieses Motivs (vgl. Frenzel 1999: 484-498).

Eine narrative Anreicherung des autobiographischen Substrats wird in Wir Kuckuckskinder überdeutlich. Ein signifikantes Nebenmotiv bildet die literarische Schnittstelle zwischen kommunikativem und kulturellem Gedächtnis: das Buch mit dem Titel „Geschichte“, das Sersch auswendig lernt und immer bei sich trägt. In der Funktion eines Amuletts wird das Buch zunächst intimisiert. Nach der Hinrichtung des unschuldigen Protagonisten fängt es sein Blut auf und wird zum Gral-Substitut. Mit dem impliziten Verweis auf das Blut Christi greift der Autor das Element der christlichen Ikonographie und Mystik auf (vgl. dazu Rubin 2001: 94-97 und Knust u.a. 2010). Das Besondere an den autobiographiebasierten Texten Pristavkins besteht darin, dass er die sozialrealistisch präformierte Motivkonstante der Waisenheimkinder neu interpretiert und sie konsequent mit der Opferrolle verschränkt. Das Psychogramm der Kinder macht sie zu modernen Picaros, ihr Leidensweg und ihr Tod zu sozialistischen Märtyrerfiguren. Dabei bringt Pristavkin zwei kollidierende Modelle in Verbindung: den Märtyrer als spätantike und vor allem christliche Variante eines gewaltlosen Helden und das Motiv des antiheroischen Schelms, dessen Figur seit je der Literatur als Instrument der Satire dient (vgl. dazu Frenzel 1999: 631-642).

Der Kurzroman steht in einer engen Beziehung zur symbolischen Form des kollektiven Gedächtnisses, und zwar zur religiösen Tradition, in der die Bibel als zentraler Text fungiert. Die religiöse Konnotation findet nicht nur auf der bildlichen Ebene statt: An die Stelle des in der Sowjetunion sehr erfolgreichen sentimentalistischen Abenteuerromans von Mayne Read The Headless Horseman (1860), den der junge Pristavkin als Souvenir aus einem brennenden Haus gerettet hatte (vgl. Pristavkin 2006: 478), werden die Entstehungsmythen Mesopotamiens gesetzt. Diese haben eine frappierende Ähnlichkeit mit den alttestamentlichen 
Schriften (vgl. El-Radhi 1999: 26-31). Mit der Biographie von Sargon, dem Herrscher von Akkad und Begründer der ersten sumerischen Dynastie, wird der Aussetzungsmythos des Knaben Moses rekapituliert und das biographische Schicksal, keine Eltern zu haben, problematisiert. Das Motiv der Sintflut als strafende oder kathartische Erneuerung bringt der Protagonist mit der drakonischen Disziplin in sowjetischen Kinderheimen in Verbindung: „Bestimmt herrschte auf dem Floß ein Spezialregime, sonst wären sie ja alle ersoffen“(Pristavkin 1990: 82). Das zeitlich Nächste der aktuellen Probleme wird durch das zeitlich Fernste erklärt.

Mehrfach klingt als Leitmotiv das „Klagelied zur Beruhigung des Herzens“ an, das zum Untertitel des Romans gehört. Dabei handelt es sich um ein altmesopotamisches Klagelied (1400-1200 v. Chr.) gegen die Götter, das spirituellkonzeptionelle Nähe und thematische Verwandtschaft mit dem Buch Hiob aufweist (vgl. El-Radhi 1999: 47-52). Ähnlich wie beim biblischen Hiob wird das Wort Klage bei Pristavkin in einem dreifachen Sinn von Weh-Klagen bzw. Sich-Beklagen und An-Klage verwendet. Während die Klage sich an eine göttliche Instanz richtet und die Trauer und das Gedenken in die Sprache übersetzt, steht die Anklage als Beschuldigung im Kontext der Täterermittlung und Verurteilung (vgl. hierzu Weigel 2000: 127-132). Nicht nur die Totenmemoria gehört zu den zentralen Botschaften des Werkes, sondern auch die Klage über die mangelnde Empathie mit den Leidenden. Immer wieder erhebt der Ich-Erzähler literarische Anklage gegen das sowjetische Regime wegen des menschenverachtenden Umgangs mit den Waisenkindern.

Anlässlich des Kriegsendes, das sich 2005 zum sechzigsten Mal jährte, veröffentlichte Pristavkin zwei weitere Kriegserzählungen über elternlose Kinder. In Ein Waggon geht auf Reisen (Vagončik moj dal'nij) rollen im Jahre 1944 evakuierte Moskauer Waisenkinder zusammengepfercht in einem Güterwaggon durch Sibirien. Als der Wagen für mehrere Wochen abgestellt wird, eskaliert die Gewalt der Soldateska. Mit Brot gefügig gemacht, werden die hungernden Mädchen aus dem Zug von sowjetischen Offizieren missbraucht, in geistige Verwirrung oder den Selbstmord getrieben. Den sechzehnjährigen Ich-Erzähler Anton verkauft man für eine Kiste Wodka als Arbeitssklaven an einen Landwirt (vgl. dazu Leingang 2010: 55-68).

Tag der Bestrafung (Sudnyj den'), das dramaturgisch interessanteste Werk Pristavkins, ähnelt einem Kammerstück. Von zahlreichen Innenweltdarstellungen durchzogen, geißelt dieser stilistisch ungewöhnliche, multiperspektivische Roman die barbarischen Arbeitsgesetze der Stalin-Ära. Bereits für Bummelei und Verspätungen von mehr als zwanzig Minuten drohten den Arbeitern drakonische Disziplinarmaßnahmen (vgl. Hildermeier 1998: 646ff). Die Volksgerichte 
schreckten nicht einmal davor zurück, Minderjährige für Verstöße zur Zwangsarbeit zu verurteilen. Im Plot ist eine weitere biographische Reminiszenz zu finden. Als es galt, Schlachten an der Produktionsfront zu gewinnen, half der jugendliche Pristavkin einem Nachbarn, einen Baum zu fällen. Er verpasste seine Schicht, als er auf dem Markt das Brennholz gegen Lebensmittel einzutauschen versuchte. Allein das beherzte Eingreifen seines Vorgesetzten konnte ihn vor Verurteilung bewahren. ${ }^{5}$ In welchem Maß der autobiographische Kern überformt wird, lässt sich an einem der eingeflochtenen Handlungsstränge zeigen: Der eigentliche Grund für den Verstoß gegen die Arbeitsdisziplin ist die sich anbahnende Liebe zwischen dem sechzehnjährigen Akkordarbeiter und einer jungen Obstverkäuferin.

\section{Posttemporalität der Erinnerungen als Lizenz zur Fiktionalisierung?}

Für Pristavkin war es selbstverständlich, seine biographischen Erfahrungen literarisch zu verarbeiten: „Als der Krieg vorbei war, wusste ich, dass ich über meine erste Lebensperiode schreiben mußte, [...] ich hatte ein abgeschlossenes Leben hinter mir" (Pristavkin 1991: 3929). ${ }^{6}$ Wie bei den meisten Autoren der Kriegskindergeneration wird das Kriegstrauma bei ihm trotz des Mitteilungsbedürfnisses erst an der Schwelle des Alters reaktiviert, und zwar mit dem Wunsch nach selbstenthüllender Bilanz. Bis dahin hatte die emotionale Anästhesie eine psychische Betondecke gegen die anflutenden Erinnerungen und Ängste geboten. Abgesehen von dem Kurzgeschichtendebüt vermeidet es Pristavkin fast zwei Jahrzehnte, seine Erlebnisse im Krieg in Worte zu fassen. Das Trauma als körperlich eingekapselte Erfahrung versperrt sich der Versprachlichung (vgl. dazu Assmann 2012: 19-40). Gerade hochgradig belastende Erfahrungen überfordern nachhaltig die individuellen Bewältigungsmöglichkeiten und können aufgrund ihrer extremen emotionalen Intensität weder hinreichend verarbeitet noch in die eigene Identitätsstruktur eingebettet werden (vgl. Bohleber 2011: 9-16).

5 „Ich arbeitete auf der Žukovskij-Fabrik bei Ljuberzy. Mich rettete damals der Chef des Laboratoriums. Die Geschichte ging so. Mein Nachbar beschloss, auf seinem Grundstück einen Baum zu fällen. Damals kam man dafür ins Gefängnis. Deshalb musste man nachts nicht nur den kompletten Baum zersägen, sondern auch die Holzspäne und den Baumstumpf beseitigen. Für die Arbeit bekam ich einen Holzblock und ging mit ihm auf den Markt, um ihn zu verkaufen, ich hatte einen schrecklichen Hunger. Und weiter beschreibe ich die Geschichte eines Jungen, der sein Holzklötzchen nicht verkaufen kann, weil er glaubt, man gebe ihm weniger, als es tatsächlich kostet. [... ] Ja. An diese Szene erinnere ich mich sehr gut.“ In: http://www.rg.ru/2005/04/14/ pristavkin.html (Abgerufen: 24.09.2012).

6 Russische Worte, Eigennamen und Orte werden grundsätzlich in der wissenschaftlichen Transliteration wiedergegeben, die im deutschen Sprachraum üblich ist. Sofern nicht explizit anders angegeben, wurden alle Titel und Zitate aus den russischen Quellen von mir übersetzt. 
Erst nach einer langen Latenzzeit schreibt Pristavkin über seine traumatische Kindheit und Jugend. Zum Tragen kommen die Perspektive des einstigen Kindes ebenso wie der für die Metaebene der Schreibgegenwart gültige Erinnerungsfokus und Interpretationsrahmen des erwachsenen Autors mit seinen Wertvorstellungen und Sinngebungsversuchen. Es liegt auf der Hand, dass die autobiographische Identität des Erzähl-Subjekts und des Erzähl-Objekts jegliche Neutralität der Darstellungen unmöglich macht. Die Tatsache, dass die Werke in bewusster Reflexion der geschilderten Ereignisse entstanden sind, bringt ihnen die Kritik der Posttemporalität ein, d. h. der Distanz zwischen Erlebnis- und Erinnerungshorizont. Das ,Jetzt ${ }^{`}$ des Erinnerungsabrufs und das ,Damals‘ des Erlebten beleuchten die memoriale Differenz zwischen vergangener Erfahrung einerseits und fiktiv überformender, retrospektiv sinnstiftender Erinnerung andererseits. Gleichzeitig drängen sich die Fragen nach der Beschaffenheit von Erinnerungen auf, die als konstruiert, standortgebunden und durch ständige Modifikationen deformiert gelten. Oft steht das objektiv Stattgefundene dem subjektiv Gedeuteten diametral entgegen. Der Literaturwissenschaftler Richard N. Coe beschreibt die Problematik der Kindheitsdarstellung wie folgt (Coe 1984: 76):

The Childhood [sic!] raises specific problems in an acute form: reliability (or arbitrary unreliability) of memory, the question of the validity of a purely subjective view of the truth, when there is no possibility of cross-checking and the conflict between the raw material of unplanned, haphazardly accumulated experience and the structural requirements of literary form.

Im Gegensatz zur internen Fokalisierung, bei der das Vergangene aus den Augen des erlebenden Ich reflektiert wird, gibt die externe Fokalisierung den gegenwärtigen, überblickenden Wissensstand der Erzählinstanz wieder. Intra- und intertextuelle Verweise, Reflexionen der eigenen Schreib- und Erinnerungstätigkeit sowie Kommentare des gealterten Kriegskindes, die als ,autoreferentielle Reflexionsschleife“ (Joachimsthaler 2009: 46) konstitutiv zum Akt des Erinnerns gehören, bilden ein diskursives Gegengewicht zu erzählter Vergangenheit.

Neben dem Authentizitätsanspruch des faktentreuen Erzählens mussten die autobiographischen Texte die Identität des Autobiographen enthüllen, und zwar durch die paratextuell markierte Personalunion von Autor, Erzähler und Protagonist, die sich formal im Namen manifestiert (vgl. Lejeune 1989: 232). ${ }^{7}$ Die autobiographische, Wahrheit', - die Übereinstimmung zwischen der ,Realität ${ }^{\star}$ und der subjektiven Erinnerung - die als das zentrale Kriterium galt, wurde in der

7 Dazu auch Holdenried (2000: 41-44), Wagner-Egelhaaf (2000: 58-63) sowie Waldmann (2000: 16-34), zur narrativen Inszenierung des kindlichen Blicks s. die Studien von Barth (2009) und Hofmann (2010). 
Autobiographieforschung längst zugunsten einer generellen Selbstreferentialität aller literarischen Texte aufgegeben (vgl. dazu stellvertretend Gronemann 1999: 239). Die individuelle Erinnerung als Akt der Imagination gesteht den autobiographischen Texten zwangsläufig die Möglichkeit der fiktionalen Anreicherungen und Stilisierungen zu. Spätestens seit Goethes programmatischem Titel Dichtung und Wahrheit ist der hybride Charakter dieser Textsorte zwischen Fakt und Fiktion im Bewusstsein (vgl. dazu Wagner-Egelhaaf 2006a: 353-355). Folgt man einem Diktum von Holdenried (2000: 23f), die sich auf Foucault stützt, so kann jeder Text autobiographisch gelesen werden. Fiktionale Überformungen konkretisieren und modellieren das Vergangene nach einem expliziten oder latenten Interpretationsmuster. Mit der Dezentralisierung des modernen Subjekts werden auch Subjektivität und Identität obsolet, die ja konstitutiv zum autobiographischen Schreiben gehören. Die Fülle an autobiographisch gefärbten Texten und die zahlreichen Stellungnahmen Pristavkins, die durch Werkkommentar zur Autorbiographie werden, erlauben es, die Fiktionalisierung seines Lebens zu rekonstruieren.

\section{Moderate Fiktionalisierung als literarische Therapie}

Die (therapeutische) Rekonstruktion der eigenen Identität pendelt bei Pristavkin zwischen der Inszenierung einer identitätsstiftenden Gemeinschaft, den Kommentaren aus der Retrospektive, den Narrationen aus kindlich-naiver Sicht, der Erfindung eines alter ego mit neuem Namen sowie der fiktionalen Überformung des autobiographischen Kerns (vgl. dazu Lathey 1999: 78). Dass sich das literarische Euvre Pristavkins aus einem einzigen Erinnerungsreservoir speist, zeigt die Tatsache, dass seine Werke untereinander nicht nur durch das Figurenensemble verklammert sind, sondern auch durch zahlreiche Szenen, prägnante Formulierungen, Anekdoten, Gedicht- und Liedfetzen. Versatzstückartig werden sie immer wieder aufgerufen, ohne einer sprachlichen oder inhaltlichen Veränderung zu unterliegen. Die strukturellen Wiederholungen als ästhetisches Prinzip stützen ein in sich stimmiges Identitätskonzept der Erzählinstanz. Das Beharren auf einer Version der Vergangenheit suggeriert wiederum einen stabilen und verifizierbaren Charakter der Erinnerungen, der in den letzten Jahrzehnten sowohl von den Neurowissenschaften, als auch den Literaturwissenschaften radikal in Frage gestellt wurde.

Wie bereits angesprochen, handelt es sich beim Gesamtwerk Pristavkins um einen typischen Fall für die Verwobenheit von Fiktion und Autobiographie, um das vieldiskutierte Problem der autobiographischen Fiktionalität (vgl. dazu Holdenried 2000: 38-44). Der so genannte „moderate Fiktionalisierungsschub“ (Ewers 2005: 
114) überführt das pränarrativ Biographische des Autors durch nachträglich hinzuphantasierte Entlastungen und Selbstfiktionalisierungen mehrmals in eine „Wunschbiographie“ (Holdenried 2000: 40), einen autobiographiebasierten Text mit starkem Hang zur Fiktion. Der Akt des Fingierens verquickt Imaginäres und Reales, Erinnertes und Vergessenes. ${ }^{8}$

\section{Archäologische Gedächtnismetapher und Konstruktionen der Erinnerungen}

Angesichts des absehbaren Endes vom Erfahrungsgedächtnis fühlt sich Pristavkin dem Erinnerungsgebot verpflichtet und inszeniert seine Werke als Medien intergenerationeller Kommunikation: „Dies bedeutet, dass diese Welt, die ich in mir trage, mit mir vergeht. Deshalb schreibe ich auch. Aber nicht willentlich, sondern nur, wenn die Erinnerungen kommen. “(Pristavkin 2005: 399) Sein Erinnerungskonzept veranschaulicht er mithilfe einer Gedächtnismetapher, welche die Verknüpfung von Erinnerung und Identität symbolisch verdichtet (vgl. Assmann 1999: 149-171). Die Schichtungsstruktur macht seine Erinnerungen zu einem archäologischen Schauplatz, an dem das Verdrängte von damals nach und nach freigelegt werden kann (Pristavkin 2005: 412):

Der Krieg hält mich in seiner Umarmung fest umklammert und lässt mich nicht mal in Erinnerungen los. [...] Aber wenn man sich die Vergangenheit als eine archäologische Ausgrabung vorstellt - denn das menschliche Gedächtnis ist im Grunde genommen eine Ausgrabung, nur ungeordneter - würde die Vorkriegsschicht heller und filigraner aussehen, gefüllt mit unterschiedlichen Kleinigkeiten, wie etwa mit bunten Splittern von Flaschen, kleinen Münzen, Federchen, Papierchen von billigen Süßigkeiten. Die Kriegsschicht - das sind Bombensplitter, Lebensmittelkarten, Kartoffelbeete mitten in der Stadt entlang der Eisenbahnlinie „Rjazanka“ und Fenster, die mit Papierbändern in Kreuzform verklebt wurden, damit die Glassplitter von der Druckwelle nicht ins Haus reinfliegen.

Aufgrund der Erstmaligkeitserlebnisse bescheinigt Pristavkin der kindlichen Erinnerungsfähigkeit eine stärkere Einprägungskraft. Die Intensität des sinnlichen Erlebens von Details spielt für ihn eine zentrale Rolle: „Die Sommerbläue des Himmels, der heiße Staub, der die Fußsohlen verbrennt, und die gelblich abgeblühten, süßlich schmeckenden Akazienkelche neben dem Haus, die sich

8 Ewers notiert dazu: „Die fiktionale Überformung der eigenen Kindheits- und Jugenderlebnisse kann [...] dazu dienen, sich eine partiell andere Biographie zu erschreiben. Für Jahrgänge mit teilweise stark belastenden Kindheits- und Jugenderfahrungen stellt dies eine nicht geringere Verlockung dar: Erlittene Entbehrungen, Erniedrigungen, schmerzhafte Verluste von primären Bezugspersonen, Schutzlosigkeits- und Verlassenheitstrauma - all diese bedrückenden Erlebnisse können durch nachträglich hinzuphantasierte Entlastungsfaktoren virtuell gewissermaßen geheilt werden" (Ewers 2005: 118). 
nun in winzige Schoten verwachsen, aus denen später Lockpfeifchen gemacht werden“ (Pristavkin 2005: 379ff). Demnach werden die Erinnerungen nicht als trügerische, wandelbare Gebilde, sondern als statische Konstrukte inszeniert. Der autobiographische Erzähler schreibt seinem Gedächtnis exakte Qualität zu, die an die dauerhafte Speicherkapazität von externen Gedächtnismedien heranreicht: „An manche erinnere ich mich aufgrund der merkwürdigen Ausschließlichkeit des kindlichen Gedächtnisses, ich erinnere mich nicht nur an ihr Gesicht, sondern auch an ihre Nach- und Vornamen" (Pristavkin 1995: 23). Das Insistieren auf der Untrüglichkeit seiner Erinnerungen führt zur Vernachlässigung solcher Verfahren, die Mechanismen narrativer Identitätsbildung in Frage stellen könnten.

In den letzten Jahren unterlag das wissenschaftliche Paradigma des Autobiographischen gravierenden Veränderungen. Unter anderem rückte der Schreibprozess in den Fokus (vgl. Wagner-Egelhaaf 2006b: 88). Auch im Spätwerk Pristavkins lässt sich diese Wendung zur Reflexivität des Erinnerten beobachten. Das Herantasten an die traumatischen Kindheitserinnerungen ist geprägt von Verzögerungsmomenten. Am Beispiel einer Unterbrechung des Erzählflusses beleuchtet der autobiographische Erzähler die Funktionsweisen von Erinnerungen (Pristavkin 2005: 397):

Plötzlich bemerkte ich bei mir eine seltsame Angewohnheit. Wenn ich mich vorsichtig an die Linie herantaste, hinter welcher der Krieg anfängt, sogar schon den Fuß über die Linie hebe, zucke ich zusammen und kehre zurück in die Kindheit vor dem Krieg, über die noch nicht alles erzählt, von der noch nicht alles gezeigt wurde. Vielleicht noch nicht alles fertig erlebt und gelebt ist. Es ist keine Angst vor einem neuen Thema, das die Vergangenheit vernichtet, zerstört, es ist eher ein Aberglaube, dass ich, nachdem ich diese schicksalhafte Grenze überschritten habe, nicht mehr zurückkehren und unbekümmert-naiv sein kann.

Dieser Kommentar, in dem das erzählende Ich seine unmittelbare emotionale Beteiligung darstellt, führt das identitätsdestabilisierende Potential von Erinnerungen vor Augen. Darüber hinaus offenbart die angesprochene Meidungstaktik eine starke Kontrolle, die das sich erinnernde Ich auf den Erinnerungsprozess und die Ereignischronologie ausübt. Zu Recht problematisiert der Literaturwissenschaftler Jürgen Joachimsthaler die nostalgische Suche nach der verlorenen Zeit (Joachimsthaler 2009: 37):

Das sich erinnernde Ich entfernt sich freilich mit seiner Erinnerungsarbeit, mit dem angestrengten Haschen nach dem scheu Enthuschenden, das nur in ungestörter Freiheit durch die Imagination zu gleiten vermag, oft nur immer weiter von dem, was es auf diese Weise vergeblich einzufangen versucht. Es müht sich von sich hinfort und entgleitet sich, indem es sich sucht - und löst damit oft nur noch verstärkte Erinnerungswerkelei aus, vertreibt sich (als erinnertes) damit aber immer nur noch weiter vor sich selbst (als sich erinnerndes). 
„Nichts ist erfunden oder erdacht, aber alles literarisch konstruiert“, behauptete Anatolij Pristavkin in einem Interview (Pristavkin 1991: 3929). ${ }^{9}$ Dieses Zitat belegt nicht nur die selbstreflexive Schriftlichkeit und den Konstruktionscharakter autobiographischer Texte (vgl. Wagner-Egelhaaf 2000: 39ff), sondern weist auf die hybride Form dieser Textsorte hin, die sich zwischen historischem und ästhetischem Diskurs verorten lässt (vgl. dazu Gasser 2011: 16). Der explizit-dokumentarische Gestus in Rjazanka und in Der erste Tag - der letzte Tag der Schöpfung bietet eine detailversessene Inventur sowjetischer Stimmungen. Gespickt mit Radioübertragungen, patriotischen Aufrufen, zahlreichen Frontliedern, kursierenden Gerüchten, sprichwörtlich gewordenen Durchhalteparolen Stalins geben Pristavkins Romane unter dem Firnis individueller Identitätsstiftung die epochenspezifische Mentalität wieder. Der reminiszierende Erzähler beschreibt ausführlich zahlreiche Photographien, die als Gedächtnisstützen zu fortexistierenden Abdrücken eines vergangenen Augenblicks gerinnen. Briefe, Notizen und amtliche Dokumente werden als vergegenständlichte Erinnerungen in den Text montiert, was wiederum eine starke Authentizitätsillusion aufbaut. Dank ihrer autobiographischen Färbung erheben seine Werke den Anspruch, authentische Erfahrungsberichte zu sein.

\section{Autobiographische Versuche}

Während sein autobiographisch inspiriertes Euvre eher eine mit Wirklichkeitssplittern durchsetzte Fiktion darstellt, zählt Rjazanka zu den autobiographischsten unter seinen Werken. Rjazanka, das einzige vom Autor als Roman bezeichnete Werk, blickt auf eine lange Entstehungsgeschichte zurück: Fast zwei Jahrzehnte, 1963 bis 1984, arbeitet Pristavkin daran und nimmt erst 1990 die Endredaktion vor. Die Kurzgeschichten aus seinem Debützyklus Die schwierige Kindheit fließen in den ersten Teil ein. In einem Vorortzug sitzend, lässt der IchErzähler Anatolij eigene Kindheit und Jugend kaleidoskopartig Revue passieren. Er wird zu einem ,Vergangenheitstouristen'. Wie die Trojka in dem Abenteuerroman von Nikolaj Gogol' Die toten Seelen bildet der Vorortzug Rjazanka den Rahmen für die gesamte Handlung. Die Stationen auf der Zugfahrt ${ }^{10}$ sind visuelle Koordinaten, über die erinnert wird. ${ }^{11}$ Sie fungieren als cues, welche die Wiederbelebung

9 Ähnlich äußert sich James E. Young (1988: 17): „All writing, all composition, is construction. We do not imitate the world, we construct versions of it. There is no mimesis, only poeisis. No recording. Only construction.” (Hervor. im Orig.)

${ }^{10}$ Das Leitmotiv der Zugfahrt bzw. des Eisenbahnwagens als wichtigstes Transportmittel der Kriegszeit greift Pristavkin erneut in Mein kleiner Waggon in der Ferne auf. Der titelgebende Waggon - ein Bild für die Irrfahrten der Menschen während des Krieges - wird zum entmaterialisierten Erinnerungsort, der durch Mobilität seine Indexikalität einbüßt.

${ }^{11}$ Zum Motiv der Lebensreise als traditionsreichem (auto-)biographischen Topos s. Christen 1999. 
individueller Erinnerungen in Gang setzen und sich unmittelbar auf die IchKonstruktion des Erzählers auswirken.

In Rjazanka verwendet Pristavkin ein mehrschichtiges Verfahren: Die erste Erzählebene ist die Zugfahrt in seinen Geburtsort Ljuberzy bei Moskau, die zweite sind die Kindheits- und Jugenderinnerungen an den Alltag der Kriegsund Nachkriegszeit, die dritte ist die Metaebene der Erinnerungsgegenwart. Dieses Mäandern zwischen dem Einst des Erlebten und dem Jetzt des Erinnerns macht die Funktionsweise von Erinnerungen anschaulich. Die Tatsache, dass der Erzähler oft aus dem Kreis seiner individuellen Erinnerungen hinaustritt, um die Lebensgeschichten seiner Eltern oder das tragische Schicksal seiner Freunde darzustellen, führt vor Augen, dass der Akt des autobiographischen Erzählens sich nicht monologisch, sondern in einem Spannungsfeld der Dialogizität entfaltet.

Die eingangs erwähnte Erzählung über den Zweiten Weltkrieg Der erste Tagder letzte Tag der Schöpfung ist ein Destillat des überbordenden autobiographischen Wissens, in dem die verstreuten Mosaiksteinchen aus den bisherigen Werken zu einem Ganzen zusammengefügt werden. Gebündelt in titellose Kapitel paraphrasiert Pristavkin seine zuvor geschriebene Rjazanka - den wichtigsten Prätext -, indem er jene Wendepunkte seiner Kindheit minutiös rekapituliert, die sich für immer in sein Gedächtnis einbrannten, wie die Beerdigung seiner Mutter und die Rückkehr des Vaters von der Front. Die Mehrfachthematisierung identitätsrelevanter Ereignisse sichert ferner die Authentizität ab, welche die subjektiv empfundene Glaubwürdigkeit untermauern soll. Dass diese Autobiographie im Dienste einer Selbstvergegenwärtigung und mit ihr verbundenen Rechtfertigungsgestus steht, bestätigt die Tatsache, dass die Erinnerungen viele apologetische Momente in Gang setzen und dadurch zur Beichte asozialen Verhaltens werden. Seine Schuldhypothek reicht von den antisemitischen Parolen, die er den Erwachsenen achtlos nachspricht, über den Diebstahl eines Elfenbeindöschens aus dem Zimmer einer Erzieherin bis hin zu weiteren kleinkriminellen Machenschaften.

Die Bindung an das kulturelle Erbe, die Pristavkin zunächst mit den Referenzen auf den literarischen Kanon stützt, wird durch die Anknüpfung an die christliche Tradition noch verstärkt. Im Gegensatz zu dem atheistischen Tenor früherer Werke hat sein Alterswerk eine starke religiöse Grundierung, die im Dienste der Autoritätssicherung ebenso wie der Identitäts-(Re-)Konstruktion steht. Auf eine große Nähe des sich erinnernden Ich zur Religion lässt sich aus intertextuellen Bezügen zur Bibel schließen. Eingerahmt in zahlreiche Gebete und alttestamentarische Zitate werden christliche Tugenden, wie Bewährung im Leiden oder jesuanischer Verzicht auf Vergeltung, mehrfach angesprochen. Das christliche Bekennertum zeigt den Einfluss des außerliterarischen Diskurses der 
Entstehungszeit: Nach dem Kollaps der Sowjetunion avancierte die russischorthodoxe Kirche als Hüterin des Vaterlandsbegriffs zum Garanten der nationalen und kulturellen Identität.

\section{Fazit}

Die hochgradig autobiographischen Werke Anatolij Pristavkins stellen ein singuläres Beispiel dar, wie im Rahmen obsessiv anmutender Aufarbeitung der Kriegskindheit eine autobiographische Serie entstehen kann. Dabei dienen psychologische Bedürfnisse nach Selbstvergewisserung und Selbstverstehen als Triebfeder des Schreibens. Mit einer einmaligen Aufzeichnung der Kriegserinnerungen war den langwelligen Erschütterungen jener Zeit nicht beizukommen. Die Landkarte der eigenen Erinnerungen wollte Pristavkin immer genauer vermessen. Dank der Fortsetzungsstruktur der Romane mit kleinmaschig werdenden Erinnerungen treten persönliche Entwicklung des Autors und aktuelle außerliterarische Diskurse zutage. Die expliziten Autobiographien Rjazanka und Der erste Tag - der letzte Tag der Schöpfung zeigen die variierende autobiographische Dichte der Werke an. Dabei werden nicht nur die Selektions- und Konstruktionsmechanismen der individuellen Erinnerungen aufgedeckt, sondern die nachjustierende Identitätsstiftung offen gelegt.

\section{Literatur}

\section{Primärliteratur}

Pristavkin, Anatolij. 1962. Malen 'kie rasskazy. Moskva: Sovetskij pisatel'.

Pristavkin, Anatolij. 1982. Soldat i malčik. Povesti. Moskva: Sovetskij pisatel'.

Pristawkin, Anatolij. 1988. Über Nacht eine goldene Wolke. Aus dem Russ. von Th. Reschke. München: Knaus.

Pristawkin, Anatolij. 1990. Wir Kuckuckskinder. Aus dem. Russ. von Th. Reschke. Berlin: Volk und Welt.

Pristawkin, Anatolij. 1992. Schlief ein goldenes Wölkchen. Aus dem Russ. von Thomas Reschke. Erste vollst. dt. Ausgabe. Berlin: Volk und Welt.

Pristavkin, Anatolij. 1995. Kukušata. Izbrannaja proza. Moskva: Kvadrat.

Pristavkin, Anatolij. 2006. Vagončik moj dal'nij. Povesti. Moskva: Izdatel'svo Ėksmo (Russkaja klassika XX veka).

Pristawkin, Anatoli. 2012. Ein Waggon geht auf Reisen. Erzählung. Aus dem Russ. von Thomas Reschke. Zürich: Offizin.

\section{Sekundärliteratur}

Assmann, Aleida. 1999. Erinnerungsräume: Formen und Wandlungen des kulturellen Gedächtnisses. München: Beck.

Assmann, Aleida. 2012. „Über Gewalt, Trauma und den Begriff der Zeugenschaft“. In Die Zukunft der Erinnerung und der Holocaust, hrsg. von Hartmann, Geoffrey und Aleida Assmann, 9-40. Konstanz: Konstanz University Press. 
Barth, Mechthild. 2009. Mit den Augen des Kindes: Narrative Inszenierung des kindlichen Blicks im 20. Jahrhundert. Heidelberg: Winter.

Bohleber, Werner. 2011. „Traumata - Transgenerationelle Weitergabe und Geschichtsbewusstsein“. In Vererbte Wunden. Traumata des Zweiten Weltkrieges die Folgen für die Familie, Gesellschaft und Kultur, hrsg. von Hondrich, Curt, 9-24. Lengerich: Pabst Science Publishers.

Christen, Matthias. 1999. "to the end of the line": Zu Formgeschichte und Semantik der Lebensreise. München: Fink.

Coe, Richard N. 1984. When the Gras Was Taller: Autobiography and the Experience of Childhood. New Haven: Yale University Press.

El-Radhi, Falah. 1999. Babel und die Bibel: mesopotamische Mythen und biblische Märchen. Frankfurt a. M.: R. G. Fischer.

Ewers, Hans-Heino. 2005. „Zwischen geschichtlicher Belehrung und autobiographischer Erinnerungsarbeit. Zeitgeschichtliche Kinder- und Jugendliteratur von Autorinnen und Autoren der Generation der Kriegs- und Nachkriegskinder". In Geschichte und Geschichten: Die Kinder- und Jugendliteratur und das kulturelle und politische Gedächtnis, hrsg. von v. Glasenapp, Gabriele und Gisela Wilkeding, 97-129. Frankfurt a. M.: Lang.

Ewers, Hans-Heino. 2012. Literatur für Kinder und Jugendliche: Eine Einführung in Grundbegriffe der Kinder- und Jugendliteraturforschung. 2. überarbeitete und aktualisierte Auflage. Paderborn: Fink.

Finck, Almut. 1995. „Subjektbegriff und Autorschaft: Zur Theorie und Geschichte der Autobiographie“. In Einführung in die Literaturwissenschaft, hrsg. von Pechlivanos, Miltos u.a., 283-294. Stuttgart: Metzler.

Frenzel, Elisabeth. 1999. Motive der Weltiteratur: Ein Lexikon dichtungsgeschichtlicher Längsschnitte. 5. überarbeitete und ergänzte Auflage. Stuttgart: Kröner.

Gasser, Peter. 2011. „Autobiographie und Autofiktion. Einige begriffskritische Bemerkungen“. In: „,.., ,, all diese fingierten, notieren, in meinem Kopf ungefähr wieder zusammengesetzten Ich “, hrsg. von Pellin Elio und Ulrich Weber, 13-18. Göttingen: Wallstein/Chronos.

Gronemann, Claudia. 1999. „,Autofiction' und das Ich in der Signifikantenkette: Zur literarischen Konstitution des autobiographischen Subjekts bei Serge Doubrovsky“. Poetica 31: 237-262.

Hildermeier, Manfred. 1998. Geschichte der Sowjetunion 1917-1991: Entstehung und Niedergang des ersten sozialistischen Staates. München: Beck.

Hofmann, Regina. 2010. Der kindliche Ich-Erzähler in der modernen Kinderliteratur: Eine erzähltheoretische Analyse mit Blick auf aktuelle Kinderromane. Frankfurt a. M: Lang.

Holdenried, Michaela. 2000. Autobiographie. Stuttgart: Reclam.

Joachimsthaler, Jürgen. 2009. „Die memoriale Differenz. Erinnertes und sich erinnerndes Ich“. In Gedächtnis und kultureller Wandel: Erinnerndes Schreiben - Perspektiven und Kontroversen, hrsg. von Klinger, Judith und Gerhard Wolf, 33-52. Tübingen: Niemeyer.

Jureit, Ulrike. 2010. Gefühlte Opfer: Illusionen der Vergangenheitsbewältigung. Stuttgart: Klett-Cotta.

Kardin, V. [Emil Vladimirovič]. 2000. "Nas bilo dvoe: brat i ja...": Zur Erzählung von Anatolij Pristavkin „Nočevala tučka zolotaja.“ <http://www.lib.ru/PROZA/ PRISTAWKIN/tuchka.txt_.txt> (Abgerufen: 24.09.2012)

Kelly, Catriona. 2007. Children's World: Growing Up in Russia, 1890-1991. New Haven/ London: Yale University Press. 
Knight, Rebecca. 2009. "Representations of Soviet Childhood in Post-Soviet Texts by Ludmila Ulitskaja and Nina Gabrielian”. Modern Language Review 103 (3): 790-808.

Knust, Christine und Dominik Groß (Hgg.). 2010. Blut: Der ganz besondere Saft in Medizin, Literatur, Geschichte und Kultur. Kassel: kassel university press.

Lathey, Gillian. 1999. The Impossible Legacy: Identity and Purpose in Autobiographical Children's Literature Set in the Third Reich and the Second World War. Bern: Lang.

Leingang, Oxane. 2010. „Tschetschenen, Wolgadeutsche, Kinder der ,Volksfeinde' - Die Demontage der Feindbilder in autobiographisch fundierten Jugendromanen von Anatolij Pristawkin“. In Kinder- und Jugendbuchforschung 2009/2010, hrsg. von Dolle-Weinkauff, Bernd u.a., 55-68. Frankfurt a. M.: Lang.

Lejeune, Philippe. 1989. „Der autobiographische Pakt“. In Die Autobiographie: Zur Form und Geschichte einer literarischen Gattung, hrsg. von Niggl, Günther, 214-257. Darmstadt: Wissenschaftliche Buchhandlung.

Pristavkin, Anatolij. 1991. „Ich habe nichts als Erinnerung!“. Das Gespräch führte Barbara Scharioth. In Börsenblatt des deutschen Buchhandels (8. November), 3929-2932.

Pristavkin, Anatolij. 2005. „Prošu proščenija: Sovmestit' v sebe pisatelja i činovnika ne vozmožno“. Das Gespräch führte Igor Ševelev. Rossijskaja gazeta - Federal'nyj vypusk 3745 (14. April ). <http://www.rg.ru/2005/04/14/pristavkin.html> (Abgerufen: 24.09.2012).

Pütz, Peter. 2004. Wiederholung als ästhetisches Prinzip. Bielefeld: Aisthesis.

Rubin, Miri. 2001. „Blut: Opfer und Erlösung in der christlichen Ikonographie“. In Blut, Kunst, Macht, Politik, Pathologie, hrsg. von Bradburne, James, 94-97. München: Prestel.

Snyder, Timothy. 2011. Bloodlands - Europa zwischen Hitler und Stalin. Aus. d. Engl. von Martin Richter. München: Beck.

Wagner-Egelhaaf, Martina. 2000. Autobiographie. Stuttgart: Metzler.

Wagner-Egelhaaf, Martina. 2006a. „Autofiktion oder: Autobiographie nach der Autobiographie: Goethe - Barthes - Özdamar". In Autobiographisches Schreiben in der deutschsprachigen Gegenwartsliteratur. Band I: Grenzen der Identität und der Fiktionalität, hrsg. von Breuer, Ulrich und Beatrice Sandberg, 353-368. München: Juricium.

Wagner-Egelhaaf, Martina. 2006b.,„Autofiktion-Theorie und Praxis des autobiographischen Schreibens“. In Schreiben im Kontext von Schule, Universität, Beruf und Lebensalltag, hrsg. von Berning, Johannes u.a., 80-101. Berlin: Lit.

Waldmann, Günter. 2000. Autobiographisches als literarisches Schreiben: kritische Theorie, moderne Erzählformen und -modelle, literarische Möglichkeiten eigenen autobiographischen Schreibens. Baltmannsweiler: Schneider Hohengehren.

Warneke, Martina. 2000. Die Rezeption russischer Gegenwartsprosa in der Bundesrepublik 1976-1995: Versuch einer Chronologie. Hamburg: Kovac.

Weigel, Sigrid. 2000. „Zeugnis und Zeugenschaft, Klage und Anklage. Die Geste des Bezeugens in der Differenz von, identity politics', juristischen und historiographischen Diskurs“. In Zeugnis und Zeugenschaft (Jahrbuch des Einsteins Forum 1999), 111135. Berlin: Akademie Verlag.

Young, James E. 1988. Writing and Rewriting the Holocaust: Narrative and the Consequence of Interpretation. Bloomington/Indianapolis: Indiana University Press. 


\section{Oxane Leingang}

Goethe University, Frankfurt - Institute for Children's Literature Research

Sveučilište „Johann Wolfgang Goethe“ Frankfurt - Institut za istraživanje dječje književnosti

\section{The Fictionalization of Wartime Childhood in Young Adult Novels by Anatolij Pristavkin}

"I was created by war", writes Anatolij Pristavkin in his last autobiographical povest - a Russian term denoting a medium length prose narrative - characteristically entitled The First Day - the Last Day of Creation (Pervyj den' - poslednij den' tvorenija). As he lost his mother to tuberculosis at the age of ten, and knew his father to be fighting at the front, the war became the context of his socialisation: Pristavkin spent his childhood in various orphanages, and was in 1944 evacuated to North Caucasus, where he was one of the few of Muscovite orphans to escape massacre. Pristavkin's large-scale literary project is focused on this traumatic biography. He approaches his childhood with increasing introspection in six short novels and countless short stories. The gaps in his inconsistent traumatic memories are bridged with repetitions which disguise the construction of an intrafictional reality and guarantee narrative coherence. But at the same time they obscure the constructed nature of such memories, which are conditioned by context and an individual perspective. Thus breaking the 'autobiographical pact', Pristavkin transforms what he does not want to express about his own life into imaginative moments of relief, into so-called "ideal autobiographies". The paper demonstrates how Pristavkin depicts and elaborates his traumatic war memories in his young adult novels.

Keywords: autobiography, Pristavkin, wartime childhood, Word War II, young adult fiction, orphans

\section{Fikcionalizacija vlastitoga ratnoga djetinjstva u romanima za mladež Anatolija Pristavkina}

„Stvorio me rat“, bilježi Anatolij Pristavkin u autobiografskom djelu Prvi dan - posljednji dan postanka (Pervyj den' - poslednij den' tvorenija) u kojemu je rat uistinu postao njegov socijalizacijski kontekst. Pristavkinova je majka umrla od tuberkuloze 1941., kada je autor imao deset godina, a otac se borio na fronti. Djetinjstvo i mladost proveo je u različitim dječjim domovima. Zajedno sa stotinama djece evakuiran je 1944. godine na Sjeverni Kavkaz gdje se s tek nekolicinom moskovske siročadi uspio spasiti od masakra. Žalostan zbog svojega djetinjstva, autor započinje veliki književni projekt. U šest autobiografskih, vrlo detaljnih kratkih romana, ali $i$ u mnogobrojnim kratkim pričama, približava se traumi svojega ratnoga djetinjstva. Budući da u tekst ne ugrađuje autobiografske signale uvriježene za 'autobiografski ugovor' te se koristi mogućnošću „pomicanja u smjeru odmjerene fikcionalizacije“, Pristavkin dijelove svoje biografije, koje ne želi posredovati, preoblikuje u imaginativne trenutke olakšanja, $u$,„željene biografije“. U radu se, na temelju Pristavkinovih autobiografskih djela Rjazanka $i$ Prvi dan - posljednji dan postanka te $s$ osnoncem na njegova osobna svjedočanstva, istražuje način na koji Anatolij Pristavkin u djelima za mladež uprizoruje svoja osobna traumatska prisjećanja na Drugi svjetski rat.

Ključne riječi: autobiografija, Pristavkin, ratno djetinjstvo, Drugi svjetski rat, romani za mladež, siročad 